\title{
Design of an Adaptive Predistorter for Solid State Power Amplifier in Wireless OFDM Systems
}

\author{
Rui J. P. de Figueiredo, ${ }^{1}$ Lin Fang, ${ }^{1}$ and Byung Moo Lee ${ }^{2}$ \\ ${ }^{1}$ Laboratory for Intelligent Signal Processing and Communications, Department of Electrical Engineering and Computer Science, \\ University of California, Irvine, CA 92697-2625, USA \\ ${ }^{2}$ Central R\&D Laboratory, Korea Telecom (KT), Seoul 137-792, South Korea
}

Correspondence should be addressed to Byung Moo Lee, blee@kt.com

Received 21 April 2009; Accepted 31 July 2009

Recommended by Paul Cristea

Orthogonal frequency division multiplexing (OFDM) is a powerful modulation choice for wideband wireless communication systems. However, its high peak-to-average power ratio greatly limits the high power amplifier (HPA) power efficiency. Here, we present the design of an adaptive predistorter to compensate the distortion caused by the HPA. Specifically, we deal with the implementation issue of the proposed predistorter in Lee and de Figueiredo's work (2006). The performance improvement by predistorter is verified by both floating-point simulation and fixed-point simulation, where the latter includes the distortion effects from the hardware. The bit widths for OFDM signals, ADC, and DAC are evaluated, and the bit width of 10 is shown to be sufficient for the hardware design.

Copyright (c) 2009 Rui J. P. de Figueiredo et al. This is an open access article distributed under the Creative Commons Attribution License, which permits unrestricted use, distribution, and reproduction in any medium, provided the original work is properly cited.

\section{Introduction}

Othogonal frequency division multiplexing (OFDM) has attracted a lot of attention from the modern wireless communication community, because of its several desirable features for high-speed data transmission. In OFDM, a broadband signal is broken down into multiple narrowband subcarriers and implemented efficiently by using the IFFT algorithm [1]. OFDM advantages include: lower intersymbol interference, efficient use of frequency/spectrum through the use of different modulation/coding across subcarriers, and superior narrowband interference suppression capabilities. However, we need to consider the practical hardware limitations of low-cost RF and mixed signal devices when designing OFDM systems for broadband wireless data transmission. One of them is the high power amplifier (HPA) linearity and dynamic range, since OFDM signals have higher peak to average power ratios (PAPRs) than other high-performance modulations, and thus extra care is required. One of the most promising approaches to the mitigation of the PAPR problem is to use a predistorter applied to the OFDM signal prior to its entry into HPA. Its purpose is to compensate the nonlinearity of the HPA and improve the system performance. Many researchers have been investigating OFDM predistorter schemes [2-5]. However, all of these techniques are based on a general approximation form for the nonlinear system, rather than exploiting specific forms gleaned from physical device considerations. Due to this reason, we proposed a closedform predistorter represented by a few parameters [6].

In this paper, we will apply Rapp's SSPA model [7] for HPA devices and show the implementation plan of the predistorter which was introduced in [6]. Furthermore, this paper also provides the design of a tracking algorithm for the case in which the practical HPA is unknown and varying. Finally, the simulation results are presented to investigate the performance improvement from predistorter, and study the distortion effects caused by saturation, overflow, and quantization with different number of bit widths, since the bit width of OFDM baseband (OFDM BB) and DAC/ADC is limited by cost and design constraints in real systems.

\section{System Description}

As shown in the block diagram of Figure 1, the proposed OFDM predistorter is placed after the OFDM baseband 
(BB) block to compensate for the degradation function in HPA.

In Figure 1, $r, q$, and $u$ are amplitudes of output of the BB, predistorter, and HPA block, respectively. From the normalized Rapp's SSPA model [7], we have

$$
u[r]=\frac{r}{\left(1+\left(r / A_{0}\right)^{2 p}\right)^{1 / 2 p}},
$$

where $u$ is the HPA output amplitude, $A_{0}>0$ is the maximum output amplitude from HPA, and $p>0$ is the parameter which controls the smoothness of the transition from the linear to the saturating region. Please note that the phase distortion for HPA is very small and hence can be neglected [7], and thus, the predistorter mainly focuses on amplitude compensation. In order to compensate the nonlinearity of the HPA, using the predistorter, the HPA output $u$ is targeted to be linear to $r$. Thus,

$$
\frac{q(r)}{\left(1+\left(q(r) / A_{0}\right)^{2 p}\right)^{1 / 2 p}}=r .
$$

Thus, we can derive the following equation [6]:

$$
q(r)=\frac{r}{\left(1-\left(r / A_{0}\right)^{2 p}\right)^{1 / 2 p}}, \quad r<A_{0} .
$$

When $r \geq A_{0}$, (3) has no solution, and $q$ has to be clipped which will be explained in the following section. Figure 2 shows a compensation example of the predistorter [6]. The upper and lower lines pertain to the PD and SSPA model, respectively, and the solid line represents the compensated effect. It shows similar effect with soft envelop limiter.

\section{Design Architecture}

The closed form expression of predistorter output $q$ is shown in the previous section. However, the information of HPA parameters $A_{0}$ and $p$ are unknown and time varying in practice. Figure 3 shows the detailed design of the architecture of our predistorter for a real time-varying environment.

The major block in the OFDM predistorter is an LMS block to update $A_{0}$ and $p$ estimations and $q$ estimation block which will be explained next in detail. As for other supporting blocks, R/P (rectangular coordinates transfer to polar coordinates) and $\mathrm{P} / \mathrm{R}$ (polar coordinates transfer to rectangular coordinates) are employed with a 12-stage cordic algorithm. A look-up table is used to store the precalculated values for the calculation of LMS update and $q$ estimation.

3.1. LMS Update. The total period is divided into two stages: the training stage covers the start time and periodic pilot time, and the remaining time is the operation stage (see Figure 4).

During the training stage, $q$ estimation block is off, that is, $q$ is set to be equivalent to the input signal magnitude of $r$, and correspondent HPA output $u$ is known. The goal during

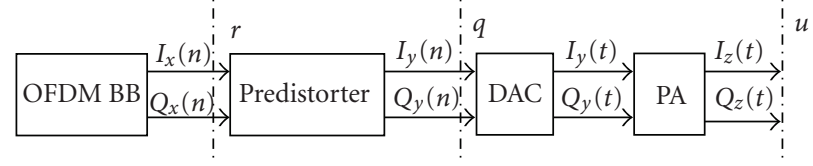

FIGURE 1: The diagram of OFDM predistorter.

this stage is to track the solution of time-varying $A_{0}$ and $p$ by LMS algorithm. We define mean square error as follows:

$$
J\left(A_{0}, p\right)=E\left(\frac{q}{\left(1+\left(q / A_{0}\right)^{2 p}\right)^{1 / 2 p}}-u\right)^{2}
$$

From the appendix, we have the expressions of $\partial J\left(A_{0}, p\right) / \partial A_{0}$ and $\partial J\left(A_{0}, p\right) / \partial p$. For LMS algorithm, expected value is replaced by instantaneous value. Therefore,

$\widehat{A}_{0}(n+1)$

$=\widehat{A}_{0}(n)-\mu_{1} \cdot\left[\left(\frac{q(n)}{\left(1+\left(q(n) / \hat{A}_{0}(n)\right)^{2 \hat{p}(n)}\right)^{1 / 2 \hat{p}^{(n)}}}-u(n)\right)\right.$

$\left.\cdot\left(\frac{q(n)}{\hat{A}_{0}(n)}\right)^{2 \hat{p}(n)+1} \cdot\left(\frac{1}{\left(\left(1+\left(q(n) / \hat{A}_{0}(n)\right)^{2 \hat{p}(n)}\right)^{1 / 2 \hat{p}^{(n)+1}}\right.}\right)\right]$,

$\hat{p}(n+1)$

$=\hat{p}(n)-\mu_{2} \cdot\left\{\left(\frac{q(n)}{\left(1+\left(q(n) / \hat{A}_{0}(n)\right)^{2 \hat{p}(n)}\right)^{1 / 2 \hat{p}^{(n)}}-u(n)}\right)\right.$

$\cdot\left(\frac{q(n)}{\left(1+\left(q(n) / \hat{A}_{0}(n)\right)^{2 \hat{p}^{(n)}}\right)^{1 / 2 \hat{p}^{(n)}}}\right)$

$\cdot\left[\left(-\frac{1}{\hat{p}(n) \cdot\left(1+\left(q(n) / \hat{A}_{0}(n)\right)^{2 \hat{p}^{(n)}}\right)}\right)\right.$

$\left.\left.\cdot \ln \left(\frac{q(n)}{\hat{A}_{0}(n)}\right) \cdot\left(\frac{q(n)}{\hat{A}_{0}(n)}\right)^{2 \hat{p}(n)}+\frac{\ln \left(1+\left(q(n) / \hat{A}_{0(n)}\right)^{2 \hat{p}(n)}\right)}{2 \hat{p}^{2}(n)^{2}}\right]\right\}$,

where $\mu_{1}$ and $\mu_{2}$ are step factors which will be defined in the following section, and the initial settings are $A_{0}(0)=1$ and $p(0)=1$.

3.2. $q$ Estimation. Figure 4 shows that after $N_{1}$ samples in training stage, LMS is turned on once per $N_{2}$ samples to update the estimates of $A_{0}$ and $p$, and turned off during 


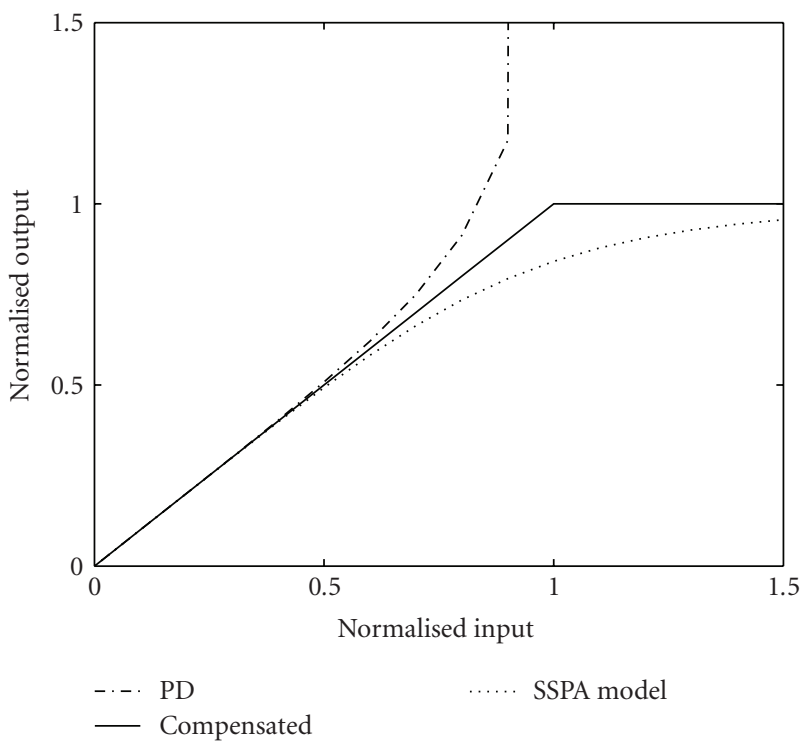

FIgURE 2: Compensation and clipping effect of SSPA with PD.

the remaining time (operation stage). Within the operation stage, predistorter is on, and $q$ estimation is calculated based on the LMS estimations of $A_{0}$ and $p$. That is,

$$
q(n)= \begin{cases}\frac{r(n)}{\left(1-\left(r(n) / \widehat{A}_{0}(n)\right)^{2 \hat{p}(n)}\right)^{1 / 2 \hat{p}^{(n)}},} & r(n)<\widehat{A}_{0}(n), \\ q_{\max }, & r(n) \geq \widehat{A}_{0}(n) .\end{cases}
$$

Please note that when $r(n) \geq \widehat{A}_{0}(n), q(n)$ is clipped to $q_{\max }$. In this paper, we set $q_{\max }=8$, which will be shown to be suitable in the numerical result session.

3.3. Look-up Table. Five sets of function results are required to be stored in the look-up table to calculate the following functions: $F_{1}(x, y)=\left[x^{2 y} /\left(1-x^{2 y}\right)\right]^{1 / 2 y}, F_{2}(x)=\ln (x)$, $F_{3}(x, y)=\ln \left[1+x^{2 y}\right], F_{4}(x, y)=\left[x^{2 y} / 1+x^{2 y}\right]^{1 / 2 y}$, and $F_{5}(x, y)=x^{2 y} / 1+x^{2 y}$.

Nonlinear quantization is applied to save table space. As for $F_{1}(x, y), y$ is quantized to $m 1 / 32$ when $33 \leq m 1 \leq 96$; $x$ is quantized to $m 2 / 32$ when $1 \leq m 2 \leq 24, m 2 / 64$ when $49 \leq m 2 \leq 54, m 2 / 128$ when $109 \leq m 2 \leq 123$, or $m 2 / 256$ when $247 \leq m 2 \leq 255$. Thus, table I requires the size of $[64 *(24+6+15+9)] \times 16=3456 \times 16$. As for $F_{2}(x)$, $x$ is quantized to $m / 512$ when $1 \leq m \leq 60, m / 256$ when $61 \leq m \leq 120, m / 64$ when $31 \leq m \leq 512$ ( $x$ will be clipped as eight if it exceeds eight). Thus, table II requires the size of $(60+60+482) \times 16=602 \times 16$. As for $F_{3}(x, y), y$ is quantized to $m 1 / 32$ when $33 \leq m 1 \leq 96 ; x$ is quantized to $m 2 / 32$ when $1 \leq m 2 \leq 31, m 2 / 16$ when $16 \leq m 2 \leq 31$, $m 2 / 8$ when $16 \leq m 2 \leq 64$. Thus, table III requires the size of $[64 *(31+16+49)] \times 16=6144 \times 16$. As for $F_{4}(x, y)$, $y$ is quantized to $m 1 / 32$ when $33 \leq m 1 \leq 96 ; x$ is quantized to $m 2 / 64$ when $1 \leq m 2 \leq 63, m 2 / 32$ when $32 \leq m 2 \leq 63$, $m 2 / 16$ when $32 \leq m 2 \leq 47, m 2 / 8$ when $24 \leq m 2 \leq 64$. Thus, table IV requires the size of $[64 *(63+32+16+41)] \times$
$16=9728 \times 16$. As for $F_{5}(x, y), y$ is quantized to $m 1 / 32$ when $33 \leq m 1 \leq 96 ; x$ is quantized to $m 2 / 32$ when $1 \leq m 2 \leq 63$, $m 2 / 8$ when $16 \leq m 2 \leq 64$. Thus, table $\mathrm{V}$ requires the size of $[64 *(63+49)] \times 16=6944 \times 16$. The total size for all of these five look-up tables is $26874 \times 16$ whose area is less than $0.5 \mathrm{~mm}^{2}$ for CMOS18.

3.4. Complexity Evaluation. Based on the precalculated parameters, (5) can be expressed as

$$
\begin{aligned}
\widehat{A}_{0}(n+1)=\hat{A}_{0}(n)-\mu_{1} \\
\cdot\left\{\left[\hat{A}_{0}(n) \cdot F_{4}\left(\frac{q(n)}{\hat{A}_{0}(n)}, \hat{p}(n)\right)-u(n)\right]\right. \\
\left.\cdot F_{4}\left(\frac{q(n)}{\widehat{A}_{0}(n)}, \hat{p}(n)\right) \cdot F_{4}\left(\frac{q(n)}{\widehat{A}_{0}(n)}, \hat{p}(n)\right)\right\},
\end{aligned}
$$

$$
\begin{aligned}
& \hat{p}(n+1)=\hat{p}(n)-\mu_{2} \\
& \cdot\left\{\left[\hat{A}_{0}(n) \cdot F_{4}\left(\frac{q(n)}{\hat{A}_{0}(n)}, \hat{p}(n)\right)-u(n)\right]\right. \\
& \cdot \hat{A}_{0}(n) \cdot F_{4}\left(\frac{q(n)}{\hat{A}_{0}(n)}, \hat{p}(n)\right) \\
& \cdot\left[-\frac{F_{4}\left(q(n) / \hat{A}_{0}(n), \hat{p}(n)\right)}{\hat{p}(n)} \cdot F_{2}\left(\frac{q(n)}{\hat{A}_{0}(n)}\right)\right. \\
&\left.\left.+\frac{F_{3}\left(q(n) / \hat{A}_{0}(n), \hat{p}(n)\right)}{2 \hat{p}^{2}(n)}\right]\right\} .
\end{aligned}
$$

After HPA parameters $A_{0}$ and $p$ estimation, the $q$ is estimated by

$$
\hat{q}(n)=\widehat{A}_{0}(n) \cdot F_{1}\left(\frac{q(n)}{\widehat{A}_{0}(n)}, \hat{p}(n)\right) .
$$

Therefore, the complexity in total includes 5 addition/subtracitons and 15 multiplications which are relative low.

\section{Numerical Results}

From (1), HPA results in a highly nonlinear situation with high input amplitude, and small distortions vice versa. Therefore, a relative level of power back off is required to reduce HPA distortion. Here, we define input back-off (IBO) as

$$
\mathrm{IBO}=10 \log _{10}\left(\frac{A_{0}^{2}}{P_{\text {in }}}\right),
$$

where $P_{\text {in }}$ is input average power of OFDM signal. Next, we will perform the algorithmic level and hardware level (fixedpoint) simulations, while the latter include all the distortion effects in hardware such as round-off error and coefficient quantization. 


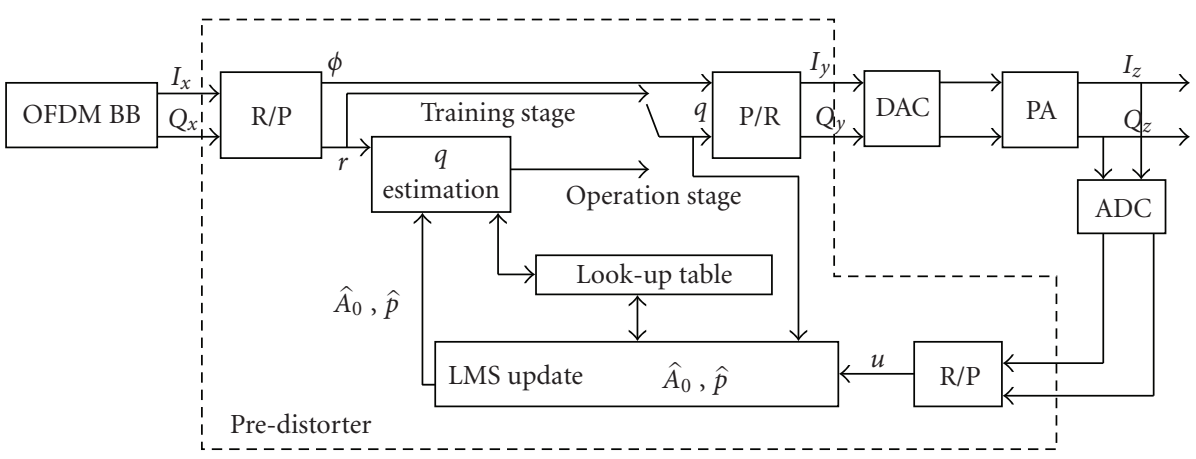

FIGURE 3: Adaptive OFDM predistorter structure.

\begin{tabular}{|c|c|c|c|c|}
\hline $\begin{array}{c}\text { Training } \\
\text { stage } \\
\text { LMS on }\end{array}$ & $\begin{array}{c}\text { Operation } \\
\text { stage } q \\
\text { estimation on }\end{array}$ & $\begin{array}{c}\text { Training } \\
\text { stage } \\
\text { LMS on }\end{array}$ & $\begin{array}{c}\text { Operation } \\
\text { stage } q \\
\text { estimation on }\end{array}$ & $\begin{array}{c}\text { Training } \\
\text { stage } \\
\text { LMS on }\end{array}$ \\
\hline$\leftarrow N_{1} T_{s} \rightarrow$ & $N_{2} T_{s} \longrightarrow \leftarrow T_{s} \rightarrow$ & $-N_{2} T_{s} \longrightarrow$ & $*$
\end{tabular}$T_{s} \rightarrow-$

FIGURE 4: predistorter function stage.

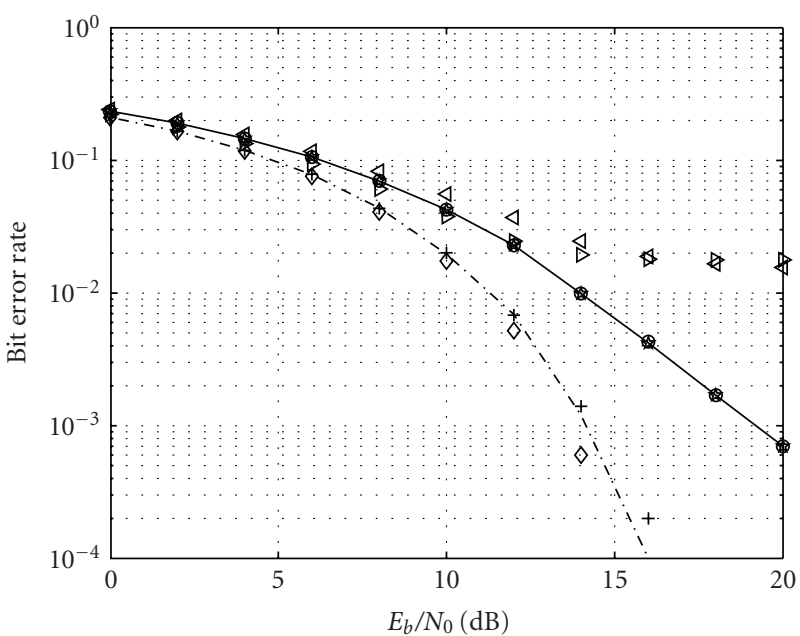

\ Float point without predistorter

$\diamond \quad$ Float point with predistorter

- 12 bits without predistorter

-.- 12 bits with predistorter

- 10 bits without predistorter

+10 bits with predistorter

$\triangleleft 8$ bits without predistorter

$\triangleright \quad 8$ bits with predistorter

FIgURE 5: Bit error rate versus $E_{b} / N_{0}$.

We set the simulation parameters as follows.

(i) 16QAM modulated OFDM signal with 64-point IFFT are studied.

(ii) The average input back-off power is $6.375 \mathrm{~dB}$, if not being mentioned.

(iii) The start training sequence is employed with a length of $N_{1}=160$ training samples, and every training sample per $N_{2}=16$ OFDM symbols is applied to the following sequence. (iv) $A_{0}$ and $p$ are both assumed to be Gaussian random numbers with mean of 1 and variance of 0.0025 .

(v) Step factor $\mu_{1}=1.5$ in training stage and 0.5 in the operation stage, while $\mu_{2}$ is set to be as much as six times of $\mu_{1}$.

(vi) The bit width of OFDM output $I_{x}, Q_{x}$, the bit width of DAC input $I_{y}, Q_{y}$, and ADC output are evaluated, since the former is limited by the area cost, and the latter is limited by DAC/ADC design.

(vii) The channel is assumed as AWGN with variance of $\eta_{0} / 2$.

From Figure 5, it shows that the bit width of 10 is recommended for OFDM BB output, DAC input, and ADC output, since there is not much improvement to increase bit width beyond 10 . The proposed implementation plan of predistorter is shown to improve system performance even including degradation effect from hardware.

\section{Conclusion}

In this paper, we have provided an implementation plan of the proposed predistorter in [6] to compensate the nonlinear distortion of SSPA. We used an LMS algorithm for time-varying environment, which we have shown to be capable of tracking SSPA parameters. Finally, a fixedpoint simulation including hardware degradation factor was performed to verify the superior performance of the proposed implementation scheme.

\section{Appendix}

\section{A. Derivative of $J\left(A_{0}, p\right)$ w.r.t $A_{0}$}

$$
\begin{aligned}
\frac{\partial J\left(A_{0}, p\right)}{\partial A_{0}}= & \frac{\partial E\left[q /\left(1+\left(q / A_{0}\right)^{2 p}\right)^{1 / 2 p}-u\right]^{2}}{\partial A_{0}} \\
=2 E\left[\left(\frac{q}{\left.\left(1+\left(q / A_{0}\right)^{2 p}\right)^{1 / 2 p}-u\right) \cdot\left(-\frac{q}{2 p}\right)}\right.\right. & \cdot\left[1+\left(q / A_{0}\right)^{2 p}\right]^{-1 / 2 p-1} \cdot q^{2 p} \\
& \left.\cdot(-2 p) \cdot\left(A_{0}\right)^{-2 p-1}\right]
\end{aligned}
$$




$$
\begin{aligned}
& =2 E\left[\left(\frac{q}{\left(1+\left(q / A_{0}\right)^{2 p}\right)^{1 / 2 p}}-u\right) \cdot\left(\frac{q}{A_{0}}\right)^{2 p+1}\right. \\
& \left.\cdot\left(\frac{1}{\left(1+\left(q / A_{0}\right)^{2 p}\right)^{1 / 2 p+1}}\right)\right] \text {. }
\end{aligned}
$$

\section{B. Derivative of $J\left(A_{0}, p\right)$ w.r.t $p$}

$$
\begin{aligned}
& \frac{\partial J\left(A_{0}, p\right)}{\partial p} \\
& =\frac{\partial E\left\{\left[q /\left(1+\left(q / A_{0}\right)^{2 p}\right)^{1 / 2 p}-u\right]^{2}\right\}}{\partial p} \\
& =2 E\left\{\left[\frac{q}{\left(1+\left(q / A_{0}\right)^{2 p}\right)^{1 / 2 p}}-u\right] \cdot \frac{\partial\left[q /\left(1+\left(q / A_{0}\right)^{2 p}\right)^{1 / 2 p}\right]}{\partial p}\right\} \\
& =2 E\left\{\left[\frac{q}{\left(1+\left(q / A_{0}\right)^{2 p}\right)^{1 / 2 p}}-u\right] \cdot q\right. \\
& \cdot \exp \left[\left(-\frac{1}{2 p}\right) \cdot \ln \left(1+\left(\frac{q}{A_{0}}\right)^{2 p}\right)\right] \\
& \left.\cdot \frac{\partial\left[(-1 / 2 p) \cdot \ln \left(1+\left(q / A_{0}\right)^{2 p}\right)\right]}{\partial p}\right\} \\
& =2 E\left\{\left[\frac{q}{\left(1+\left(q / A_{0}\right)^{2 p}\right)^{1 / 2 p}}-u\right] \cdot q \cdot\left[1+\left(\frac{q}{A_{0}}\right)^{2 p}\right]^{-1 / 2 p}\right. \\
& \cdot\left[\left(-\frac{1}{2 p}\right) \cdot \frac{\partial\left[\ln \left(1+\left(q / A_{0}\right)^{2 p}\right)\right]}{\partial p}\right. \\
& \left.\left.+\ln \left(1+\left(\frac{q}{A_{0}}\right)^{2 p}\right) \cdot \frac{\partial(-1 / 2 p)}{\partial p}\right]\right\} \\
& =2 E\left\{\left[\frac{q}{\left(1+\left(q / A_{0}\right)^{2 p}\right)^{1 / 2 p}}-u\right] \cdot q \cdot\left[1+\left(\frac{q}{A_{0}}\right)^{2 p}\right]^{-1 / 2 p}\right. \\
& \cdot\left[\left(-\frac{1}{2 p}\right) \cdot \frac{1}{\left(1+\left(q / A_{0}\right)^{2 p}\right)}\right. \\
& \left.\left.\cdot \frac{\partial\left[\left(q / A_{0}\right)^{2 p}\right]}{\partial p}+\ln \left(1+\left(\frac{q}{A_{0}}\right)^{2 p}\right) \cdot \frac{1}{2 p^{2}}\right]\right\} \\
& =2 E\left\{\left[\frac{q}{\left(1+\left(q / A_{0}\right)^{2 p}\right)^{1 / 2 p}}-u\right] \cdot q \cdot\left[1+\left(\frac{q}{A_{0}}\right)^{2 p}\right]^{-1 / 2 p}\right. \\
& \cdot\left[\left(-\frac{1}{2 p}\right) \cdot \frac{1}{\left(1+\left(q / A_{0}\right)^{2 p}\right)} \cdot \frac{\partial\left[\exp \left(2 p \cdot \ln \left(q / A_{0}\right)\right)\right]}{\partial p}\right. \\
& \left.\left.+\ln \left(1+\left(\frac{q}{A_{0}}\right)^{2 p}\right) \cdot \frac{1}{2 p^{2}}\right]\right\}
\end{aligned}
$$

$$
\begin{aligned}
& =2 E\left\{\left[\frac{q}{\left(1+\left(q / A_{0}\right)^{2 p}\right)^{1 / 2 p}}-u\right] \cdot q \cdot\left[1+\left(\frac{q}{A_{0}}\right)^{2 p}\right]^{-1 / 2 p}\right. \\
& \cdot\left[\left(-\frac{1}{2 p}\right) \cdot \frac{q}{\left(1+\left(q / A_{0}\right)^{2 p}\right)} \cdot \exp \left(2 p \cdot \ln \left(\frac{q}{A_{0}}\right)\right)\right. \\
& \left.\left.\frac{\partial\left[\left(2 p \cdot \ln \left(q / A_{0}\right)\right)\right]}{\partial p}+\ln \left(1+\left(q / A_{0}\right)^{2 p}\right) \cdot \frac{1}{2 p^{2}}\right]\right\} \\
& =2 E\left\{\left(\frac{q}{\left(1+\left(q / A_{0}\right)^{2 p}\right)^{1 / 2 p}}-u\right) \cdot\left(\frac{q}{\left(1+\left(q / A_{0}\right)^{2 p}\right)^{1 / 2 p}}\right)\right. \\
& \cdot\left[\left(-\frac{1}{p \cdot\left(1+\left(q / A_{0}\right)^{2 p}\right)}\right) \cdot \ln \left(\frac{q}{A_{0}}\right) \cdot\left(\frac{q}{A_{0}}\right)^{2 p}\right. \\
& \left.\left.+\frac{\ln \left(1+\left(q / A_{0}\right)^{2 p}\right)}{2 p^{2}}\right]\right\} \text {. }
\end{aligned}
$$

\section{References}

[1] H. Sampath, S. Talwar, J. Tellado, V. Erceg, and A. Paulraj, "A fourth-generation MIMO-OFDM broadband wireless system: design, performance, and field trial results," IEEE Communications Magazine, vol. 40, no. 9, pp. 143-149, 2002.

[2] D.-S. Han and T. Hwang, "An adaptive pre-distorter for the compensation of HPA nonlinearity," IEEE Transactions on Broadcasting, vol. 46, no. 2, pp. 152-157, 2000.

[3] H. W. Kang, Y. S. Cho, and D. H. Youn, "On compensating nonlinear distortions of an OFDM system using an efficient adaptive predistorter," IEEE Transactions on Communications, vol. 47, no. 4, pp. 522-526, 1999.

[4] S. Chang, E. J. Powers, and J. Chung, "A Compensation scheme for nonlinear distortion in OFDM systems," in Proceedings of IEEE Global Telecommunications Conference (GLOBECOM '00), vol. 2, pp. 736-740, San Francisco, Calif, USA, NovemberDecember 2000.

[5] Y. Guo and J. R. Cavallaro, "Enhanced power efficiency of mobile OFDM radio using predistortion and postcompensation," in Proceedings of the 56th Vehicular Technology Conference (VTC '02), vol. 1, pp. 214-218, Vancouver, Canada, September 2002.

[6] B. M. Lee and R. J. P. de Figueiredo, "Adaptive predistorters for linearization of high-power amplifiers in OFDM wireless communications," Circuits, Systems, \& Signal Processing, vol. 25, no. 1, pp. 59-80, 2006.

[7] C. Rapp, "Effect of HPA-nonlinearity on 4-DPSK/OFDMsignal for a digital sound broadcasting system," in Proceedings of the 2nd European Conference on Satellite Communications, vol. 2, pp. 179-184, Liege, Belgium, October 1991. 

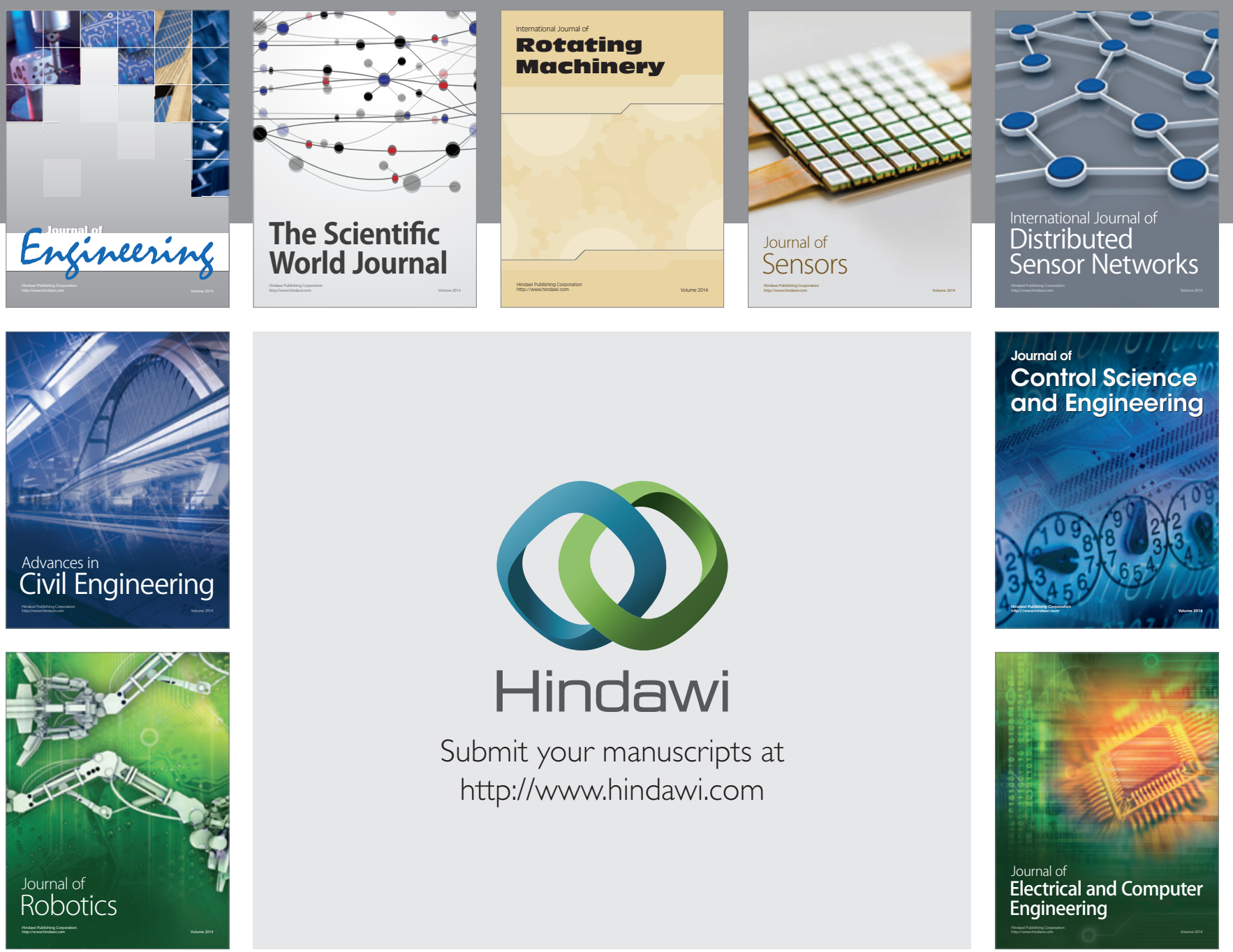

Submit your manuscripts at

http://www.hindawi.com
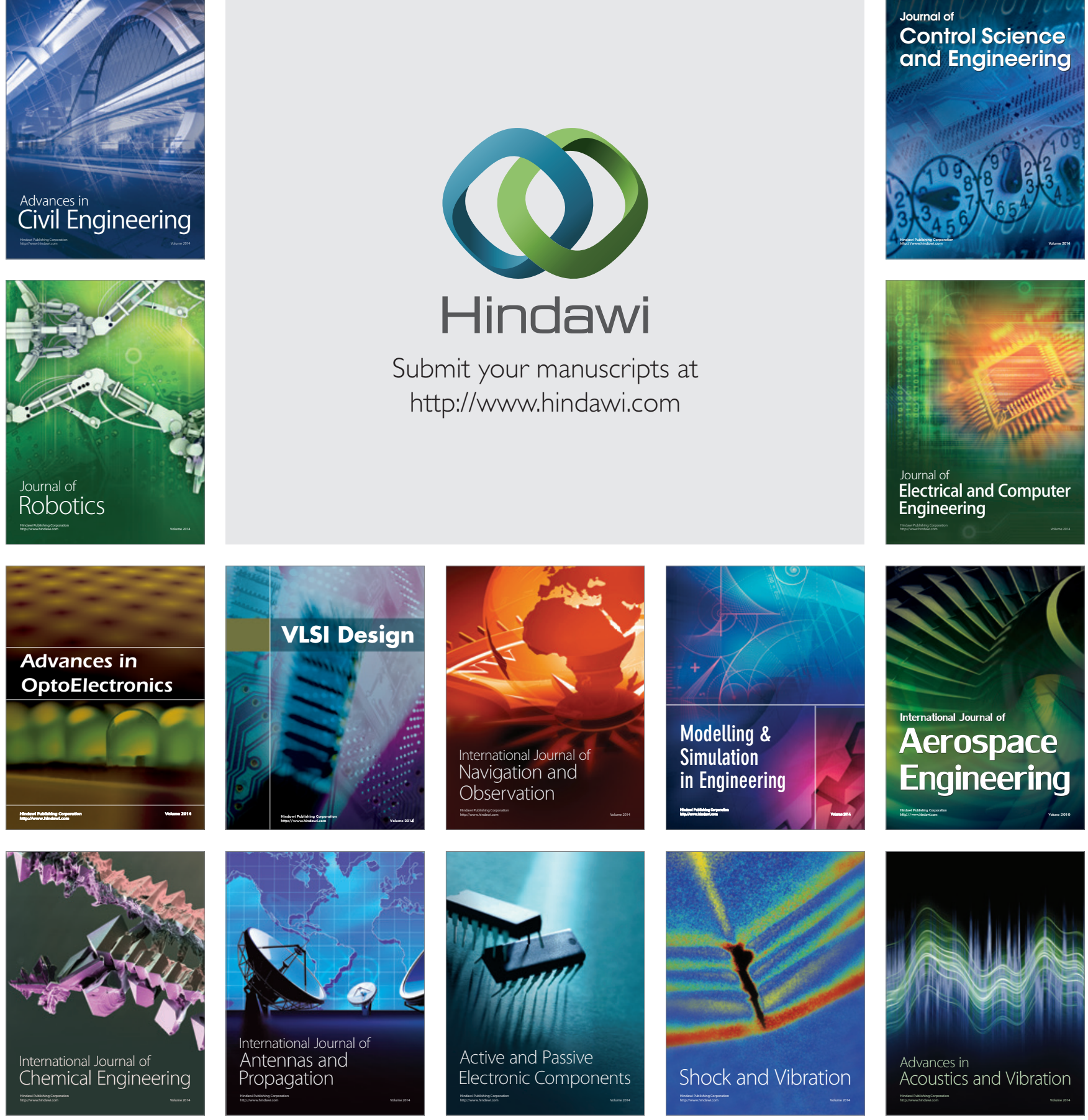